\title{
Unresectable Epitheloid Hemangioendothelioma of the Neck Region Cured by Radiotherapy
}

\author{
Elif Ayanoglu Aksoy ${ }^{\mathrm{a}, \mathrm{e}}$, Banu Atalar ${ }^{\mathrm{b}}$, Volkan Beylergil ${ }^{\mathrm{c}}$, Omer Faruk Unal ${ }^{\mathrm{d}}$
}

\begin{abstract}
Epitheloid hemangioendothelioma (EHE) is a rare vascular neoplasm which has a variable malignant potential. EHE cases have been reported in the head and neck region. This is the report of an unresectable neck EHE cured totally by radiotherapy. As EHE has a malignant potential, wide local excision is the accepted treatment of choice. If total resection is not possible, additional curative therapy modalities are needed. Invasive forms of EHE which can not be resected totally can be treated by radiotherapy.
\end{abstract}

Keywords: Epitheloid hemangioendothelioma; EHE; Neck; Supraclavicular region; Radiotherapy

\section{Introduction}

Epitheloid hemangioendothelioma (EHE) is a rare vascular neoplasm first described in 1982 by Weiss and Enzinger $[1,2]$. It has been called by various names like histiocytoid hemangioma and hemangioendothelioma because it has a variable malignant potential with a broad spectrum of occurrence between benign epithelioid hemangioma and

Manuscript accepted for publication May 5, 2011

${ }^{a}$ Acibadem Health Care Group, Maslak Hospital, Otorhinolaryngology Head \& Neck Surgery Department, Istanbul, Turkey

${ }^{\mathrm{b}}$ Acibadem University School of Medicine, Radiation Oncology

Department, Istanbul, Turkey

${ }^{c}$ Acibadem University School of Medicine, Nuclear Medicine

Department, Istanbul, Turkey

${ }^{\mathrm{d}}$ Acibadem University School of Medicine, Otorhinolaryngology Head $\&$ Neck Surgery Department, Istanbul, Turkey

${ }^{e}$ Corresponding author: ATASEHIR 38 ADA SEDEF CAD ATA 3/4 D

229 KADIKOY 3758, Istanbul, Turkey.

Email: elifayanoglu@yahoo.com

doi:10.4021/jmc216w epitheloid angiosarcoma [2-4]. EHE occurs in many tissues like liver, spleen, bone, skin, heart, soft tissues and vascular system [6]. Cases have been reported in the head and neck region [3, 4]. Oral cavity, thyroid gland, submandibular area, neck, scalp, larynx, parapharyngeal space, parotid gland and mandible were the regions of origin of these cases [3, 7-8]. Although there are reported cases of EHE in the neck region, this is the report of an unusual case of an unresectable neck EHE cured totally by radiotherapy.

\section{Case Report}

A 57-year old woman presented with a left-sided supraclavicular neck mass and neck pain. Ultrasonography of the neck region demonstrated left supraclavicular mass. Magnet-

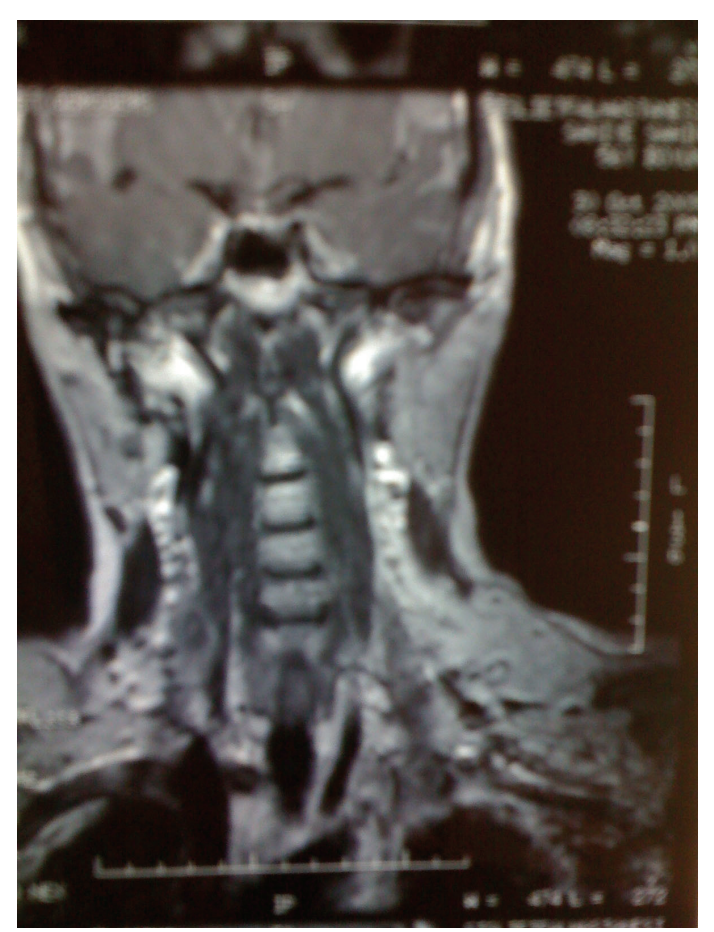

Figure 1. Sagittal view of neck MRI, without central contrast enhancement. 


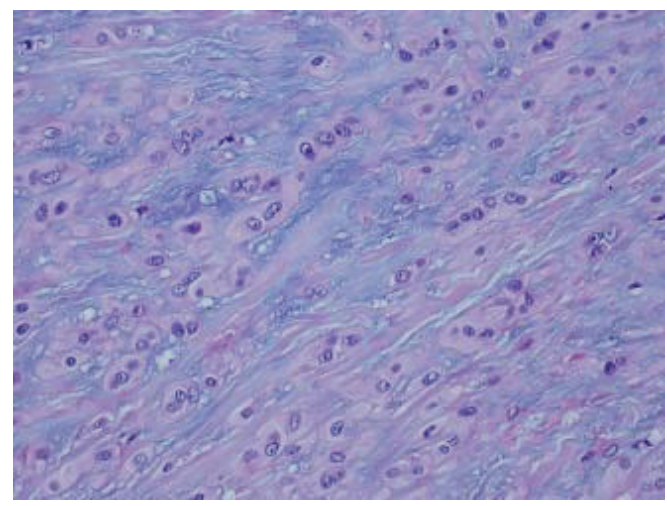

Figure 2. Spindle-shaped endothelial cells with cytoplasmic vacuolation.

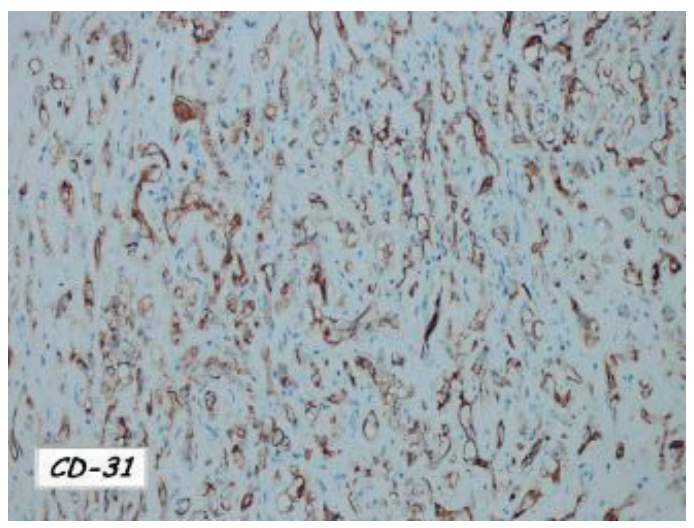

Figure 3. Endothelial cell marker CD31 positivity.

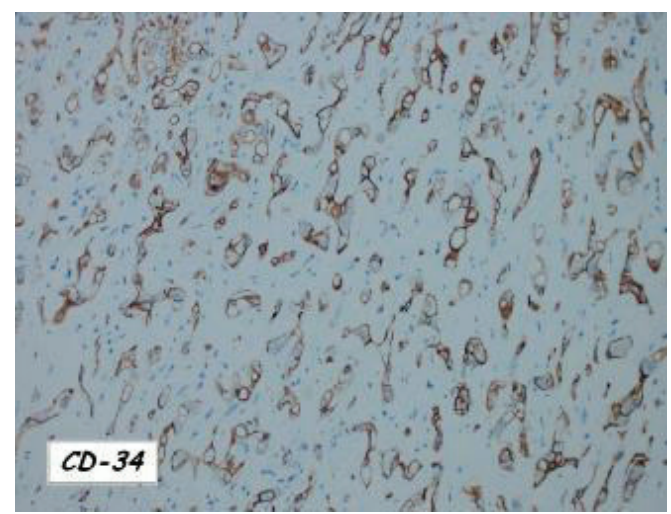

Figure 4. Endothelial cell marker CD34 positivity.

ic unresectable EHE resonance imaging of the neck showed a supraclavicular mass without central contrast enhancement (Fig. 1). Tru-cut biopsy of the supraclavicular mass was performed. Pathology report of this biopsy revealed a neoplasm rich with myxoid matrix and having epitheloid properties. Tru-cut biopsy specimen did not show high grade malignant properties, and it was hypocellular in many areas. As these morphological properties did not point out a specific diagnosis, total resection of the mass was planned. After peropera-

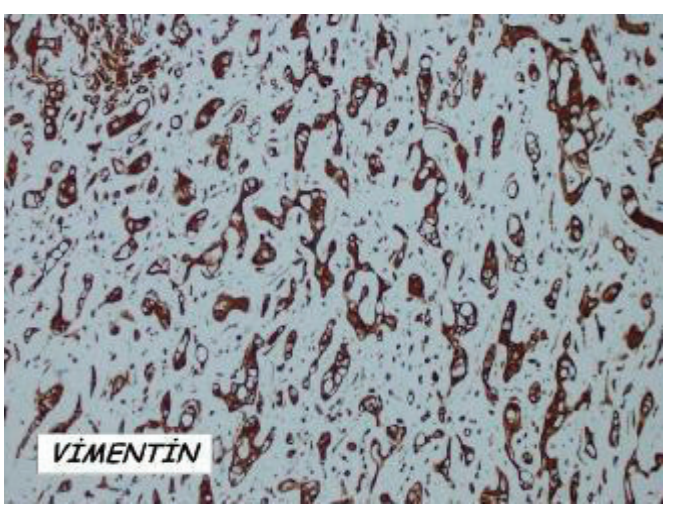

Figure 5. Vimentin.

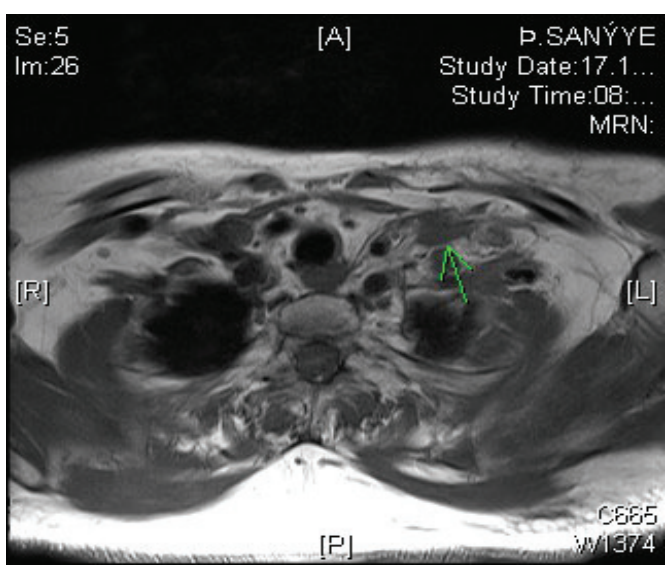

Figure 6. Axial view of neck MRI after treatment.

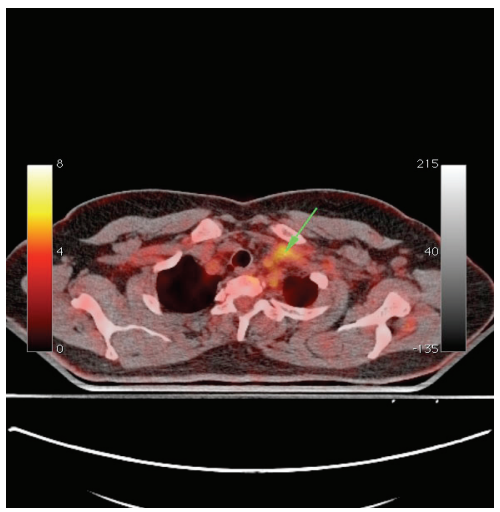

Figure 7. PET/CT before treatment.

tive evaluation of the mass, it was concluded that it was a hard, wooden, and fixed mass localized at the junction of left subclavian and jugular veins. The borders of the mass were unremarkable and the mass was involving both supra and infraclavicular regions. As the mass could not be resected surgically, incisional biopsies were taken from the mass. The pathology demonstrated EHE. Microscopic examination showed round or spindle-shaped endothelial cells with cytoplasmic vacuolisation (Fig. 2), the cytoplasm was posi- 


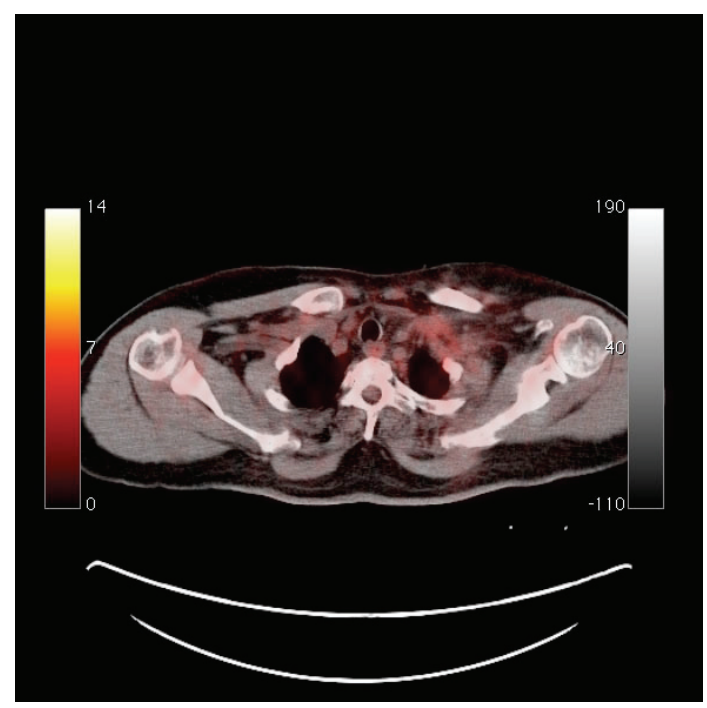

Figure 7. PET/CT after treatment, almost complete metabolic response in the left supraclavicular regions.

tive for immunohistochemical staining with the endothelial cell markers CD31, CD34 and vimentin (Fig. 3-5). After this diagnosis, for pretreatment staging a PET/CT was performed and a left supraclavicular hypermetabolic mass lesion (SUV $\max 4.05$ ) was found without distant organ metastasis. The patient was referred to radiotherapy department and treated with intensity modulated radiotherapy in liner accelerator, 6 MV photons, to a total of $60 \mathrm{~Gy}$ in 30 fractions. Radiotherapy field was planned according to MRI and PET/CT and defined as gross tumor volume which was the abnormal FDG uptake area and all suspicious areas in the MRI. The patient had complete symptomatic pain relief immediately after RT. Postradiotherapy neck MRI of the patient showed a lesion located at the left supraclavicular fossa which was isointense at $\mathrm{T} 1$ sequences with minimal contrast enhancement. The borders of the lesion were not distinct and this lesion was concluded as necrosis of the mass after RT unresectable EHE (Fig. 6). The comparative evaluation of PET/CT before and 3 months after treatment demonstrated the metabolic response of this tumor to radiotherapy. Posttreatment scans showed almost complete metabolic response in the left supraclavicular regions. Non FDG avid milimetric lymph nodes observed in the pretreatment scan were stable (Fig. 7, 8). During followup visits, the patient was free of symptoms.

\section{Discussion}

EHE is an unusual vascular tumor with borderline malignant behaviour [3,9]. This tumor is very rare in head and neck region. If EHE develops in head and neck, it usually develops at or below the level of the mandible and submandibular region is the most affected site in this area [8].
Histopathological findings of EHE are round or spindleshaped epitheloid cells with pale cytoplasm [8]. Tumor cells have vacuolisations containing red blood cells [10]. Immunohistochemical staining is a useful diagnostic tool. Endothelial cell markers (CD31, CD34 and factor VII-related antigen) aid in confirming the vascular nature of the tumor [7, 8]. In the case presented here CD31 and CD34 were positive.

Most of the cases of EHE in head and neck region reported were treated with surgical excison of the masses. As EHE has a malignant potential, wide local excision is the accepted treatment of choice. But as in the case presented here, if total resection is not possible, additional curative therapy modalities are needed. Although radiotherapy alone is reported to be rarely effective [11], in our case EHE was cured by radiotherapy mainly. The authors refusing radiotherapy propose that EHE tumor cells have slow growth rates and radiotherapy is ineffective. But EHE has a variable malignant potential and the cell growth rates may change individually. Each case of EHE should be evaluated uniquely and the treatment unresectable EHE strategy should be planned accordingly. If invasive forms of EHE can not be surgically resected, radiotherapy can be the treatment of choice.

Conflict of interest: The authors of this paper do not have any financial relationship with any organization related to this case report.

\section{References}

1. Deyrup AT, Tighiouart M, Montag AG, Weiss SW. Epithelioid hemangioendothelioma of soft tissue: a proposal for risk stratification based on 49 cases. Am J Surg Pathol. 2008;32(6):924-927.

2. Weiss SW, Enzinger FM. Epithelioid hemangioendothelioma: a vascular tumor often mistaken for a carcinoma. Cancer. 1982;50(5):970-981.

3. Lee JC, Lee BJ, Wang SG, Kim HW. Epithelioid haemangioendothelioma in the parapharyngeal space. J Laryngol Otol. 2006;120(6):505-507.

4. Erkan AN, Bal N, Kiroglu E. A case report of hemangioendothelioma of the hard palate. B-ENT. 2008;4(3):175178.

5. Moran WJ, Dobleman TJ, Bostwick DG. Epithelioid hemangioendothelioma (histiocytoid hemangioma) of the palate. Laryngoscope. 1987;97(11):1299-1302.

6. McKenzie ML. Epithelioid hemangioendothelioma of the wrist. Plast Reconstr Surg. 1985;76(5):781-783.

7. Mentzel T, Beham A, Calonje E, Katenkamp D, Fletcher $\mathrm{CD}$. Epithelioid hemangioendothelioma of skin and soft tissues: clinicopathologic and immunohistochemical study of 30 cases. Am J Surg Pathol. 1997;21(4):363374.

8. Ellis GL, Kratochvil FJ, 3rd. Epithelioid hemangioen- 
dothelioma of the head and neck: a clinicopathologic report of twelve cases. Oral Surg Oral Med Oral Pathol. 1986;61(1):61-68.

9. Amin KS, McGuff HS, Cashman SW, Otto RA. Epithelioid hemangioendothelioma of the parotid gland with atypical features. Otolaryngol Head Neck Surg. 2003;129(5):596-598.
10. Weiss SW, Ishak KG, Dail DH, Sweet DE, Enzinger FM. Epithelioid hemangioendothelioma and related lesions. Semin Diagn Pathol. 1986;3(4):259-287.

11. Pokharna RK, Garg PK, Gupta SD, Dutta U, Tandon RK. Primary epithelioid haemangioendothelioma of the liver: case report and review of the literature. J Clin Pathol. 1997;50(12):1029-1031. 